\title{
Destigmatizing Experiences of Combat Veterans Engaged in Civilian Disaster Relief: Implications for Community Transition
}

\author{
Derrick Kranke \\ June Gin \\ Rebecca Saia \\ Susan Schmitz \\ Eugenia L. Weiss \\ Aram Dobalian
}

\begin{abstract}
Combat veterans possess some attributes of differentness that may cause others in society to create stigmatized perceptions of them and devalue their skills. Direct interaction/contact allows targeted individuals-in this case, combat veterans-to demonstrate their value by dispelling negative attitudes or beliefs others may have of them. A previous study reported that the disaster relief setting facilitates contact among combat veterans and non-military civilians. This study applied Modified Labeling Theory (MLT) to assess if the stigma and labeling experience among combat veterans volunteering in disaster settings provides a nuanced understanding. Semi-structured interviews were used to assess perceptions of male Team Rubicon (TR) members $(n=9)$ who provide disaster relief in civilian settings. All participants served in combat. Data were thematically analyzed. Findings suggest 1) combat veterans were not stigmatized by their label; and 2) personal contact with civilians impacted by disaster helped TR members a) to demonstrate their value, and b) feel more optimistic about connecting with civilians in other contexts. Our findings suggest that veterans working with peer combat veterans and civilians in disaster settings provided a destigmatizing condition whereby combat veterans felt less stigmatized by others and more interpersonally connected with civilians. It is recommended that since the majority of services for veterans come from civilian community providers, it is imperative that providers be sensitive to military culture and experiences of those serving in combat to avoid further stigmatization of veterans.
\end{abstract}

Keywords: Stigma; contact; combat veterans; qualitative; Modified Labeling Theory

Public stigma contributes to veterans' struggle to reintegrate (Blais \& Renshaw, 2013; Britt et al., 2008; Danish \& Antonides, 2013; Hoge et al., 2004; Kim, Thomas, Wilk, Castro, \& Hoge, 2010; Weiss, Coll, \& Metal, 2011), particularly by reducing opportunities in education, employment, housing, and intimate relationships (Sayer, Carlson, \& Frazier, 2014). Public stigma is a psychological and social process that recognizes and distinguishes human differences, which are then linked to negative stereotypes that place labeled individuals in separate categories with undesirable characteristics (Link \& Phelan, 2001). Differentness is the antithesis of what people in society want to feel (Leavey, 2005).

Derrick Kranke, PhD, MA, is a Health Science Specialist, Veterans Emergency Management Evaluation Center (VEMEC), US Department of Veteran Affairs, North Hills, CA 91343; June Gin, PhD, is a Researcher, (VEMEC), US Department of Veteran Affairs, North Hills, CA 91343; Rebecca Saia, MA, is a Speech Pathologist, Glendale, CA 91202; Susan Schmitz, MAIDP, is a Senior Project Director, (VEMEC), US Department of Veteran Affairs, North Hills, CA 91343; Eugenia L. Weiss, PsyD, MSW, is a Clinical Associate Professor, University of Southern California, Suzanne Dworak-Peck School of Social Work, Los Angeles, CA 90089; Aram Dobalian, PhD, JD, MPH, is Director, (VEMEC), US Department of Veteran Affairs, North Hills, CA 91343. 
Combat veterans possess attributes of differentness from non-combat veterans (i.e., those veterans who have not been exposed to combat) and most others in society because military training can reinforce hyper-masculine behaviors, namely competitiveness, distrust of others, aggression, and emotional insensitivity (Ashley \& Brown, 2015; Brooks, 1999). In addition, combat veterans being exposed to or engaging in the atrocities of war can make civilians question whether or not these veterans can be trusted to not act aggressively towards others (McCann \& Pearlman, 1990; Weiss et al., 2011). In some cases, the behaviors and tendencies in adapting hyper-masculine behaviors during military service inhibit the skills necessary for resilience and effective adaptation upon returning home (Keats, 2010).

Combat veterans are inclined to interact with other combat veterans because of shared experience (Ashley \& Brown, 2015; Keats, 2010). Extant literature indicates that individuals' social network is typically made up of peers who are similar (Aboud \& Mendelson, 1996). Time spent during deployment could distance a service member from his/her social network at home, or change family dynamics upon his/her return. In contrast, time spent during combat deployment with peers can create a type of brotherhood because of enduring life-threatening situations together. As veterans return to civilian life, the connection to their combat unit may be disrupted and their social groups may expand to include more civilians. Readjustment to society may be challenging because of trust issues between combat veterans and their family and friends who do not have experiences in war contexts.

\section{Theoretical Framework}

Labeling is the identification of differences among a marginalized population which causes some in society to stigmatize and form negative conceptions of that targeted group (Corrigan \& Kleinlein, 2005). As conceptions and stereotypes of targeted groups are confirmed in individual instances (i.e., the media or personal experiences), society members may distance themselves from the targeted group because of fear for their own well-being. Ultimately, this process can lead members of society to deny meaningful social, economic, and employment opportunities to members of the targeted group. This labelling process may further contribute to self-stigmatization of those in the targeted group who may likewise adopt less than positive labels, internalizing negative beliefs about themselves and lowering their self-esteem (Corrigan, 2005).

Modified Labeling Theory (MLT; Link, Cullin, Struening, Shrout, \& Dowhenrend, 1989) posits that individuals labeled for attributes of differentness likely respond in one of two ways, either 1) the person of a different attribute does not internalize the societal label, and consequently, has no negative consequences; or 2) the target of the label applies the label to his/her self and reacts by a) being secretive about the condition to protect his/herself from discrimination and other negative effects (Goffman, 2009); and/or b) limits interaction to similar others. Limiting interaction with others outside of their group allows members of stigmatized groups to empathize with and support each other. Studies indicate that limiting interactions to those who are similar can reduce both social opportunities and the formation of meaningful relationships with others outside of the group (Corrigan, 2005; Kranke, Floersch, Townsend, \& Munson, 2010). The aforementioned reactions have 
several negative consequences: feeling shame from having to hide the condition, and/or disengagement from opportunities because he/she self-discriminates or considers themselves less than. Ultimately, the effects of stigma and labeling can have negative implications for overall health and quality of life of the targeted individual (Link et al., 1989).

\section{Contextual Factors in Responding to Labeling}

Our previous study suggested that combat veterans volunteering in disaster relief settings with Team Rubicon (TR) reported improved reintegration into society because of the interaction with other combat veterans (Kranke, Saia, Gin, Heslin, \& Dobalian, 2016). Team Rubicon is an organization comprised of over 50,000 volunteers, primarily veterans of Operation Enduring Freedom (OEF) or Operation Iraqi Freedom (OIF). Formed in 2010 by military veterans, TR deploys humanitarian aid teams to areas that are acutely affected by disasters, warfare, and other extreme events, both domestically and internationally (Disaster Response Veterans Service Organization \& Team Rubicon, n.d.). In many cases, TR members who were veterans faced similar reintegration barriers as other veterans, which helped them to normalize their shared experiences (Klein, 2015). This analysis builds upon our earlier work by examining if and how volunteering in disasters along with other combat veterans and civilians alters the experience of stigma among combat veterans. Since volunteering in disasters increases interaction with civilians, the study assessed if personal contact with (the other) civilians with no military background potentially eradicates the effects of stigma and labeling (Corrigan, 2005). Personal contact is an empirically-based intervention assumed to reduce stigma because it allows for the direct interaction with the target group. Such direct interaction could contribute to others valuing their contribution and thus disconfirming negative attitudes or beliefs others may have of them (Corrigan, 2005; Kranke \& Floersch, 2009; Weber \& Crocker, 1983). This study applied modified labeling theory (MLT), as it is instrumental in identifying the underlying assumptions of why stigmatized individuals react the way they do. Thus, what we empirically know about the stigma of a combat veteran in the context of disaster relief is limited. This analysis was guided by the following research question: What is the experience of stigma among combat veterans in interactions with civilians while volunteering in disaster relief?

\section{Methods}

\section{Sampling and Design}

The design was a cross-sectional qualitative study. The protocol was approved by the U.S. Department of Veterans Affairs Greater Los Angeles Healthcare System's Institutional Review Board. Each participant gave written informed consent before participating in the study. Researchers conducted semi-structured interviews with nine current TR members over a period of three months in 2014 at a secure and private setting on the West Coast of the United States. The participants also completed a brief selfadministered demographic questionnaire prior to each interview. 
Participants were indirectly recruited through an email message sent by the TR Program Director to the entire TR roster of volunteers in the local area $(n=155)$. The email announced the study, explained the inclusion criteria, and provided a contact number for the Principal Investigator (PI). Interested veterans called the PI to obtain more information about the study. Inclusion criteria were that the participants had to be at least 18 years of age, must be available for an in-person interview during the interview period, and must have been deployed on one mission with TR. Participation in the study did not require prior military combat experience.

\section{Sample Demographics}

All participants $(\mathrm{n}=9)$ were males who served in combat — eight in OEF and/or OIF. The average age of study participants was 36 ( $S D=11.09$; range 24-57). Five participants were in the Marine Corps, and four were in the Army. Five reported as White and four reported as Latino. Participants had been separated from the military for an average of 10.4 years $(S D=5.9$; range $6-25)$. Four were married, three were never married, and two were divorced/separated.

\section{Instrument}

The semi-structured interview collected narrative data on TR perceptions of societal attitudes and behaviors toward combat veterans, and benefits/drawbacks of interacting with other combat veterans and civilians. The term "societal attitudes and behaviors" refers to self-reports of how participants perceived opportunities and marginalization in society as they related to reintegration concerns (i.e., employment, education, housing, social networks, and intimate relationships). The term "benefits/drawbacks of interacting" refers to how the interaction with fellow combat veterans improved or reduced opportunities. We were exploring both positive and negative responses from participants about their perceptions of societal attitudes and the benefits/drawbacks of interacting with civilian others. To elicit the data, the researchers asked each respondent a series of general questions about his life after deployment before and after joining TR. The interviewers used probes as needed to elicit data on the respondent's perceptions of his daily activities, professional relationships, and physical and social environments. Each interview lasted between 60 and 90 minutes. The participants received no compensation. To ensure data safety, the transcripts were stored in a password-protected computer that could only be accessed by researchers who were given permission by the Principal Investigator of the study. Questionnaires were kept in a secure and locked cabinet.

\section{Data Analysis}

Digital audio recordings of interviews were transcribed verbatim and transferred to Atlas.ti, software specifically designed for qualitative data coding and management (Muhr, 1993). Two members of the research team conducted the thematic analysis, first by applying a priori codes, such as stigma (Kim et al., 2010), shared experience (Karp, 2006), and limiting interaction (Kranke et al., 2010; Link et al., 1989). The same two researchers also conducted open coding to identify relevant themes. We then sorted themes by shared content (i.e., "TR provides an environment where I can freely discuss my problems" and 
"I can be open with my peers in TR"). Reporting of themes was based on substantive significance (Patton, 2002) - in other words, how they informed the extant literature. In particular, we used the constant comparative method (Boeije, 2002) using constructs of MLT (Link et al., 1989) as a framework. We reported those themes that both aligned with constructs of MLT (e.g., public perceptions and limited opportunities) and those themes that added a nuanced understanding to the model (e.g., no shame or secrecy, expanding interaction beyond similar others, improved outlook). The sample size for this qualitative study was appropriate because the research question was exploratory in nature. For very detailed studies, the sample size can be in single figures (Padgett, 2008).

To establish the trustworthiness of the findings, the first and third authors created a codebook after each coded three interviews independently. They then reviewed the codes and created a master codebook that contained all of the codes from the first six interviews, and added any relevant codes to the remaining three interviews. When there was disagreement, the codes were expanded to include the characteristics that were evident across the narratives. As a cross-check, the first and third authors also conducted secondary coding of each other's analysis.

\section{Results}

Thematic analysis revealed that the label of being a "combat veteran" is frequently associated with negative stereotypes, which some veterans then internalize and apply to themselves, as MLT (Link et al., 1989) suggests. However, our findings show a more complex scenario. Participants' responses suggest that engagement in organizations like TR may be an avenue to promote interaction with civilians, thus alleviating negative stereotypes of the veterans and reducing the harmful impact of labeling. This section applies the components of the MLT to identify nuanced understandings of the stigma and labeling process by 1 ) illustrating the perceived societal stereotypes and marginalization of combat veterans in society; 2) demonstrating how combat veterans engaged in disaster relief respond to stigma and discrimination; and 3) elucidating how the context of volunteering and contact with non-military civilians in disasters can modify veterans' responses to the labeling process. See Figure 1.

\section{Before Joining TR: Perceived Stigma and Marginalization from Society}

Team Rubicon members believed that as combat veterans, they were socially stigmatized as being a burden on society. Respondents identified their perceptions of societal views of "veterans as a demographic of liabilities with a lot of problems." Many of them had internalized these societal perceptions and viewed themselves as unable to cope. One veteran said, "As vets we are encouraged to see ourselves as damaged or in need of a crutch.” These perceptions marginalize and differentiate veterans as not being able to function in civilian society. Veterans internalize these stigmatizing views into their own self-perceptions, contributing to their loss of self-confidence and feelings of being marginalized, separated, and isolated from society. 
Figure 1. TR Members' Response to Public Stigma and Labeling

Before joining TR....................After joining TR

\begin{tabular}{|c|c|c|c|c|}
\hline \multirow{3}{*}{$\begin{array}{l}\text { "Veterans are in need of } \\
\text { a crutch." } \\
\text { "Veterans are a } \\
\text { demographic of } \\
\text { liabilities." }\end{array}$} & \multicolumn{4}{|c|}{ Resist negativity of label applied to self } \\
\hline & \multirow{4}{*}{$\begin{array}{l}\text { "We're not a liability, } \\
\text { we're an asset." }\end{array}$} & \multicolumn{3}{|c|}{ No shame or secrecy about being a combat veteran } \\
\hline & & \multirow{3}{*}{$\begin{array}{l}\text { "The culture is one of } \\
\text { compassionate } \\
\text { badasses." }\end{array}$} & \multicolumn{2}{|c|}{ Connect with civilians to expand interaction } \\
\hline & & & \multirow{2}{*}{$\begin{array}{l}\text { "That's what it's about. } \\
\text {.. that moment of } \\
\text { emotional connect- } \\
\text { with the people that } \\
\text { they are directly } \\
\text { benefiting." }\end{array}$} & $\begin{array}{l}\text { Increased opportunities } \\
\text { in society }\end{array}$ \\
\hline & & & & $\begin{array}{l}\text { "“IIt's [Rubicon\} groups } \\
\text { to harness the skills of } \\
\text { vets and give them good } \\
\text { purpose and add real } \\
\text { value to society." }\end{array}$ \\
\hline
\end{tabular}


Reduced opportunities. Veterans who feel stigmatized often perceive that their veteran status limits their opportunities and that prospective employers will not want to hire them because of stereotypes about veterans' abilities. Team Rubicon members described how they perceived their employment opportunities to be limited due to the marginalization of combat veterans, and that skills built in the military were not valued in civilian life. As one TR member described, “I'd just graduated. I wasn't going to get hired any time soon because, you know, out of the 200 places I applied to I only had like maybe one response, two responses." This exhaustive effort led him to the conclusion that, "I had no talents that were valued in the market." This conclusion was devastating to him because "you did all of these great things (in the military) but now you have to start from, zero basically.”

The marginalization of combat veterans in terms of employment opportunities gave some TR members a grim outlook. For instance, one participant said, "A lot of people will come back from Iraq or Afghanistan and assume that they can never find a career or opportunity later in life that will ever sniff the value they derived from what they did overseas." Another participant remarked, "I'm never going to have as important a role as I did when I was 19 years old." They perceived that civilian life would be a letdown because their military experiences and skills were not valued at home.

Limited interaction. For TR members, social relationships with civilians, even family members, were fraught with an inability to connect or relate across the divide of their military experience and identity. One study participant mentioned they limited their interaction with non-veterans because they felt they had: "No connection, not even with my own family." Another combat veteran spoke of coming home from deployment and having a limited social network because "I didn't have many, if any, civilian friends. My whole family, I just couldn't relate to them, couldn't talk to them.” One TR member identified how those with families experience an emotional rift with their own spouse or children, who "don't understand why daddy or their husband is doing this (deployment)." Another TR member described this divide in the context of coming home and reintegrating in a college setting with non-military students who have not experienced some of the atrocities or faced the hardships that combat veterans have endured. He described his perception that society seems to value what non-veteran college students can contribute over the contributions of veterans-despite veterans' life-skills, experience, and actual service to society: "You're coming home and going to college and seeing these kids who know everything and have this happy-go-lucky attitude and you feel like they don't know everything." Veterans perception that their skills are devalued in contrast to those of civilians contributes to their sense of alienation and separation from the rest of society. These feelings may lead them to want to reduce their interaction with non-veterans.

\section{After Joining TR: Combat Veteran Response to Stigma and Labeling}

No shame or secrecy about combat veteran status. Universally, all of the TR members in this study disputed societal perceptions of veterans being a liability and in need of a crutch: "We're not a liability, we're an asset." They asserted their lack of shame or secrecy about their veteran status. One combat veteran demonstrated a valued shared identity by referring to themselves "a culture of compassionate bad-asses." They were not 
secretive about sharing their identities as combat veterans with other veterans and civilians in the disaster context because volunteering in TR offered a context for the veterans to openly discuss their experiences in combat. One TR member reported, "We all have our unique stories, and unique experiences we went through. But when we come back, it seems like we all go through the same thing . . . all our stories are the same.” It is likely that veterans in TR were motivated to expand their interaction to others in society because the shared experiences helped to reduce feelings of differentness or alienation in the larger context.

\section{Interaction with Others}

Interact with similar others. Connecting and interacting with others who share common attributes emerged as an important objective for these combat veterans. Specifically, combat veterans who served in recent conflicts (OEF/OIF) found particularly strong connections or emotional attachments with other combat veterans who served during the same time period because of a shared understanding of the close, interconnected, interdependence inherent to the daily lifestyle that they had become accustomed to during their time in combat as described by this participant:

When you're in the military, particularly when you're in combat, you experience pure, simple, collective living. We all take care of each other, we all support each other. There everyone eats the same thing, everyone sleeps in the same place, our objectives are clear. Right? Especially for a combat soldier; don't die, kill the enemy.

Team Rubicon members are able to bridge the divide between combat and non-combat veterans, branches of service, and even include civilian first-responders in creating a shared identity encompassing anyone committed to service. One respondent described how creating this large, collective, shared identity helps to reduce their sense of differentness and isolation:

You're with likeminded people; generally veterans have experienced some similar things whether the Army, Navy, Air Force, Marines, Vietnam, here and now, you're in for the same thing with the service. And a couple of the guys were just first responder test [phonetic] but they still, it's service, you know, service-oriented mentality persons.

Reshaping the other's perception. There is an emotional and/or intellectual disconnect among some civilians and combat veterans because the former cannot comprehend or fathom the values and experiences of the latter. However, one TR member did not assign blame on society for its inability to comprehend combat veterans' mentality. Rather, he articulated a broader view of the uniqueness of the veteran experience and the value that it can offer to society. He views the challenge of translating that value to civilians optimistically, as a step toward creating jobs for veterans:

We have the experience of being a relatively insignificant piece in a large, important machine. And that experience and when I'm trying to convince some of 
these companies to hire vets that's difficult to articulate, but a very important experience.

By emphasizing valued attributes, this TR member sought to reframe an identity commonly perceived as stigmatized by society as a valued identity based on shared strengths and assets: "You want someone that knows how to be a team player, take someone who's learned how to be a team player by the boot."

Connecting with the other. Team Rubicon participants reported that being able to help civilians through volunteering in TR's disaster relief enabled them to apply the strengths gained through their military background in a setting where they are uniquely qualified to assist others. This opportunity led them to feel more optimistic about their ability to connect and reintegrate with civilians in a non-military context. For instance, one TR member said, "I'm in a good place now, I'm secure, and a lot of that I attribute . . . to go and face them and talk to others like with Team Rubicon.” Another TR member described a change in his level of interaction with civilians, "Before [TR] I didn't talk to anybody. Now I interact with everybody."

In addition, several TR members described the profound experience of providing disaster relief to a civilian. Both examples demonstrate one of the first times the veteran was able to feel connected with civilians because it helped the veteran to see beyond his own struggles and focus instead on the value of human dignity in helping others in a noncombat setting: "That's what it's about . . . that moment of emotional connect-with the people that they are directly benefiting." Another TR member described an observation of a fellow combat veteran connecting with a civilian victim of a disaster: "The [TR combat veteran] person's hugging, this homeowner, this woman is just in tears, hugging this guy who's got to be sitting at 260, you know, 6-9 and he's just like, "Don't touch me, don't touch me, get away,” you know. He's about to go, he's about to have a, uh, anxiety attack. And then all of a sudden it's, it's just tears coming out."

Team Rubicon emphasizes specific strengths of combat veterans: grit, perseverance, team work, camaraderie, loyalty to fellow military combat veterans, refusal to give up in face of adversity, and continuing to serve their country: "We're all working together for that sense of pride in knowing that we've helped families out, that we've just saved lives in just a different form.” These core attributes are used by TR as tools to tackle the shared challenge of reintegration among recent OEF/OIF combat veterans, as one TR member puts it: "The common story of reintegration and isolation and lack of purpose seem to me, from my anecdotal experience, to be the common theme with this generation of veterans coming home."

All participants described TR as a vehicle for ending the cycle of stigma and marginalization associated with being a combat veteran (thus, possibly implying that engaging in TR provides a destigmatizing condition): "This is about being a support network for a very unique demographic of Americans." One member describes how TR helps veterans reintegrate by applying their distinctive skills to solve problems and demonstrating their resilience, rather than treating them as defective and in need of "fixing:" 
We are consciously attempting to set the example for a generation of veterans. . . It's not only groups to support broken vets, it's groups to harness the skills of vets and give them good purpose and add real value to society. That's good for society and that's good for the members. Everyone wants to have worth, everyone wants to contribute, particularly vets who are the type of people who took an oath to go fight a war for their neighbors.

\section{Discussion}

The application of Modified Labelling Theory (Link et al., 1989) among combat veterans who engage in civilian disaster relief provided several novel findings. There were discrepancies with some of the responses regarding the experience of stigma and labeling, and there were differences in some of the underlying assumptions of those responses. The following paragraphs describe the nuanced understandings among this population and setting.

While the MLT (Link et al., 1989) posits that the perceived stigma and label of differentness makes the targeted individual secretive about their status or label to avoid rejection, our findings suggest that while combat veterans may initially experience social stigma around their identities, they are not secretive or shameful about being labeled a combat veteran. There are several possible explanations for this. First, the stereotype by some in society is linked with attributes such as: being a hero, and fighting for the country (Brown, 1984; Woolf, 2012), or possessing hyper-masculine values (Keats, 2010)—all of which appear to be more desirable to society than those values associated with labels of mental illness or being a burden on society (Corrigan, 2005). Next, in being able to interact with civilians in a disaster context, where their military skills and identity are valued, participating in TR allowed veterans to transform their formerly devalued veteran identity among civilians into an identity that was valued and shared among those in society.

Second, although the MLT proposes that individuals with a label associated with differentness limit interaction to those they can trust or that have a similar condition to avoid discrimination or rejection (Link et al., 1989), our findings indicate that these TR members did not limit interaction out of fear of rejection or discrimination from society. Rather, our study suggests that TR members limited interaction to veterans (both combat and non-combat) because of their beliefs that civilians who have not served in the military cannot comprehend or appreciate their unique experience as combat veterans (as opposed to limiting interactions because of fear of rejection or discrimination). They believe that those who have never been in their shoes simply “don't get it” (Greden et al., 2010, p. 95).

Furthermore, our findings illustrate how volunteering in disaster relief helped to bridge the "us vs. them" gap (veterans versus civilians). The TR experience created a destigmatizing environment that fostered prolonged social interaction with civilians, which at the same time may have also made civilians realize the value of combat veterans because of the goodwill they demonstrated. Some participants described how positive interactions with civilians opened the door for job opportunities outside of TR. Ultimately, interaction with civilians increased TR members' social capital and led to additional opportunities in 
society which consequently resulted in a positive transition for these veterans into the civilian world (Kranke et al., 2016).

\section{Implications for Community Transition}

It is estimated that half of those who meet criteria for mental health conditions such as posttraumatic stress disorder or major depression do not seek services (U.S. Department of Veterans Affairs [VA], 2011). The majority of services for veterans come from civilian community providers as opposed to social workers in uniform or providers from the VA (Weiss \& Albright, 2014). Therefore, civilian providers must be sensitive to military culture and the experiences of combat veterans (Coll, Weiss, Draves, \& Dyer, 2012) in order to not further stigmatize them. From these study findings, the implications for social workers interacting with veterans whether as mental health providers or as case managers, is that the focus of interaction should be on the relational and empathic aspects of psychotherapy or service provision in order to interrupt the internalization of negative selfstigma in veterans. Taking an attachment-based perspective, a meaningful professional relationship with a civilian psychotherapist or case manager can support a positive sense of self in the client and can help shape affirming relationships with others (Badenoch, 2008) including those that have never shared the combat experience.

\section{Limitations and Future Research}

The findings of this study are not generalizable because of the small non-representative sample of male TR members. Veterans who volunteered in TR may be unique because of environmental factors and support. One must be cautious when broadly applying these findings because the study participants were highly resilient and reacted favorably to uncovering stigmatizing beliefs and societal perceptions of themselves, as well as interacting with civilians. Future work is needed to confirm the findings of this study, as well as examine the experience of stigma and labeling among combat veterans not engaged in disaster work. A broader perspective that assesses female TR members' experiences may yield different findings because of gender differences in dealing with stigma. Additionally, including a wider range of military service branches may provide a more holistic view of military culture and show potential variations of response to stigma and labeling. Representation from various ethnic or racial groups may also yield different findings as some groups have been marginalized by society on the basis of race or sexual orientation. Finally, the long term trajectory of these veterans is unknown because the study was crosssectional. Personal contact and meaningful interpersonal relationships to overcome the effects of stigma and labeling can have long-term effects. Conducting a follow-up study with this subset could yield insight about combat veterans' integrating into civilian settings, and how that transition process impacts health behaviors as well as social, employment and educational opportunities.

\section{Conclusion}

This study examined the experience of stigma and labeling among combat veterans in disaster settings by applying the MLT (Link et al., 1989). We found that combat veterans were not stigmatized by their label because the stereotypes of being a combat veteran are 
commonly linked with being a hero or fighting for their country. In addition, the combat veterans indicated they expanded their interaction beyond other veterans because the TR setting facilitated interaction among TR combat veterans and civilians victimized by disaster. Volunteering in TR helped the combat veterans bridge the gap of us vs. them. They had emotional connections and attachments with civilians in disasters and with their civilian TR teammates. Personal contact and interpersonal relationships with civilians may have had several effects. The combat veterans felt that they were of value to society again by serving their country through TR and saving lives in a different venue, thereby dispelling negative beliefs that the civilians they encountered may have had of them. These positive relationships may have also altered their sense of self and ability to connect emotionally with others. Ultimately, our findings suggest that the disaster context helped the combat veterans in TR feel more optimistic about their ability to connect with civilians in life outside of the military. This has significant implications for both engaging in psychotherapy or services with social workers as well as promoting a positive transition into civilian life post military service.

\section{References}

Aboud, F., \& Mendelson, M. (1996). Determinants of friendship selection and quality: Developmental perspectives. In W. Bukowski, A. Newcomb, \& W. Hartup (Eds.), The company they keep: Friendship in childhood \& adolescence (pp. 66-86). NY: University of Cambridge.

Ashley, W., \& Brown, J. C. (2015). The impact of combat status on veterans' attitudes toward help seeking: The hierarchy of combat elitism. Journal of Evidence-Informed Social Work, 12, 534-542. doi: https://doi.org/10.1080/15433714.2014.992695

Badenoch, B. (2008). Being a brain-wise therapist: A practical guide to neurobiology. NY: Norton.

Blais, R. K., \& Renshaw, K. D. (2013). Stigma and demographic correlates of helpseeking intentions in returning service members. Journal of Traumatic Stress, 26, 7785. doi: https://doi.org/10.1002/jts.21772

Boeije, H. (2002). A purposeful approach to the constant comparative method in the analysis of qualitative interviews. Quality and Quantity, 36, 391-409. doi: https://doi.org/10.1023/A:1020909529486

Britt, T. W., Greene-Shortridge, T. M., Brink, S., Nguyen, Q. B., Rath, J., Cox, A. L., ... \& Castro, C. A. (2008). Perceived stigma and barriers to care for psychological treatment: Implications for reactions to stressors in different contexts. Journal of Social and Clinical Psychology, 27, 317-335. doi: https://doi.org/10.1521/jscp.2008.27.4.317

Brooks, G. (1999). A few good men: Military socialization and gender role strain. Society for the Psychological Study of Men and Masculinity Bulletin, 4, 9-11.

Brown, P. C. (1984). Legacies of a war: Treatment considerations with Vietnam veterans and their families. Social Work, 29, 372-379. doi: https://doi.org/10.1093/sw/29.4.372 
Coll, J. E., Weiss, E. L., \& Draves, P., \& Dyer, D. (2012).The impact of military cultural awareness, experience, attitudes, and education on clinician self-efficacy in the treatment of veterans. Journal of International Continuing Social Work Education, 15(1), 39-48.

Corrigan, P. W. (2005). On the stigma of mental illness: Practical strategies for research and social change. Washington, DC: American Psychological Association. doi: https://doi.org/10.1037/10887-000

Corrigan, P., \& Kleinlein, P. (2005). The impact of mental illness stigma. In P. Corrigan (Ed.), On the stigma of mental illness: Practical strategies for research and social change (pp. 11-44). Washington, DC: American Psychological Association. doi: https://doi.org/10.1037/10887-001

Danish, S. J., \& Antonides, B. J. (2013). The challenges of reintegration for service members and their families. American Journal of Orthopsychiatry, 83, 550-558. doi: https://doi.org/10.1111/ajop.12054

Disaster Response Veterans Service Organization \& Team Rubicon. (n.d.). Retrieved July 10, 2015, from http://www.teamrubiconusa.org/

Goffman, E. (2009). Stigma: Notes on the management of spoiled identity. NY: Simon \& Schuster.

Greden, J. F., Valenstein, M., Spinner, J., Blow, A., Gorman, L. A., Dalack, G. W., ... \& Kees, M. (2010). Buddy-to-Buddy, a citizen soldier peer support program to counteract stigma, PTSD, depression, and suicide. Annals of the New York Academy of Sciences, 1208, 90-97. doi: https://doi.org/10.1111/j.1749-6632.2010.05719.x

Hoge, C. W., Castro, C. A., Messer, S. C., McGurk, D., Cotting, D. I., \& Koffman, R. L. (2004). Combat duty in Iraq and Afghanistan, mental health problems, and barriers to care. New England Journal of Medicine, 351, 13-22. doi: https://doi.org/10.1056/NEJMoa040603

Karp, D. A. (2006). Is it me or my meds? Living with antidepressants. Cambridge, MA: Harvard University Press. doi: https://doi.org/10.4159/9780674039339

Keats, P. A. (2010). Soldiers working internationally: Impacts of masculinity, military culture, and operational stress on cross-cultural adaptation. International Journal for the Advancement of Counselling, 32, 290-303. doi: https://doi.org/10.1007/s10447$\underline{010-9107-\mathrm{z}}$

Kim, P. Y., Thomas, J. L., Wilk, J. E., Castro, C. A., \& Hoge, C. W. (2010). Stigma, barriers to care, and use of mental health services among active duty and National Guard soldiers after combat. Psychiatric Services, 61, 582-588. doi: https://doi.org/10.1176/ps.2010.61.6.582

Klein, J. (2015). Charlie Mike: A true story of heroes who brought their mission home. NY: Simon \& Schuster. 
Kranke, D., \& Floersch, J. (2009). Mental health stigma among adolescents: Interventions for school social workers. School Social Work Journal, 34 (1), 28-42.

Kranke, D., Floersch, J., Townsend, L., \& Munson, M. (2010). Stigma experience among adolescents prescribed psychiatric medications. Children and Youth Services Review, 32(4), 496-505. doi:https://doi.org/10.1016/j.childyouth.2009.11.002

Kranke, D., Saia, R., Gin, J., Heslin, K., \& Dobalian, A. (2016). Applying an ecological strengths-based perspective to veterans' experience of reintegration into civilian life. Military Behavioral Health, 4(1), 75-81. doi: https://doi.org/10.1080/21635781.2015.1119771

Leavey, J. E. (2005). Youth experiences of living with mental health problems: Emergence, loss, adaptation and recovery (ELAR). Canadian Journal of Community Mental Health, 24, 109-126. doi: https://doi.org/10.7870/cjcmh-2005-0018

Link, B. G., Cullen, F. T., Struening, E., Shrout, P. E., \& Dohrenwend, B. P. (1989). A modified labeling theory approach to mental disorders: An empirical assessment. American Sociological Review, 54, 400-423. doi: https://doi.org/10.2307/2095613

Link, B. G., \& Phelan, J. C. (2001). Conceptualizing stigma. Annual Review of Sociology, 27, 363-385. doi: https://doi.org/10.1146/annurev.soc.27.1.363

McCann, I. L., \& Pearlman, L. A. (1990). Vicarious traumatization: A framework for understanding the psychological effects of working with victims. Journal of Traumatic Stress, 3(1), 131-149. https://doi.org/10.1007/BF00975140

Muhr, T. (1993). Atlas.ti software development. Berlin: Scientific Software Development.

Padgett, D. K. (2008). Qualitative methods in social work research (vol. 36). Los Angeles, CA: Sage.

Patton, M. Q. (2002). Two decades of developments in qualitative inquiry: A personal, experiential perspective. Qualitative Social Work, 1, 261-283. doi: https://doi.org/10.1177/1473325002001003636

Sayer, N. A., Carlson, K. F., \& Frazier, P. A. (2014). Reintegration challenges in US service members and veterans following combat deployment. Social Issues and Policy Review, 8, 33-73. doi: https://doi.org/10.1111/sipr.12001

U.S. Department of Veterans Affairs [VA]. (2011). Fact sheet: VA services for posttraumatic stress disorder. Washington, DC: Office of Patient Care Services and Office of Mental Health Services.

Weber, R., \& Crocker, J. (1983). Cognitive processes in the revision of stereotypic beliefs. Journal of Personality and Social Psychology, 45, 961-977. doi: https://doi.org/10.1037/0022-3514.45.5.961

Weiss, E. L., \& Albright, D. L. (2014). Introduction to the special issue: Mental health care for military service members, veterans and their families: Opportunities for social work. Social Work in Mental Health, 12 (5-6), 387-390. doi: https://doi.org/10.1080/15332985.2014.927408 
Weiss, E., Coll, J. E., \& Metal, M. (2011). The influence of military culture and veteran worldviews on mental health treatment: Practice implications for combat veteran help-seeking and wellness. International Journal of Health, Wellness \& Society, 1, 75-86. doi: https://doi.org/10.18848/2156-8960/CGP/v01i02/41168

Woolf, A. G. (2012). Competing narratives: Hero and PTSD stories told by male veterans returning home, Graduate Theses and Dissertations at University of South Florida.

Author note: Address correspondence to: Derrick Kranke, PhD, 16111 Plummer St. MS152, North Hills, CA 91343. Derrick.Kranke@va.gov 